\title{
Repetition Rate Tuning of an Ultrafast Ytterbium Doped Fiber Laser for Terahertz Time-Domain Spectroscopy
}

\author{
Hakan Keskin ${ }^{\mathrm{a}}$, Hakan Altan ${ }^{\mathrm{a}}$, Seydi Yavas ${ }^{\mathrm{b}}$, F. Omer Ilday ${ }^{\mathrm{b}}$, M. Emre Yagci ${ }^{\mathrm{c}}$, Ozan Aydın ${ }^{\mathrm{c}}$, Koray \\ Eken $^{\mathrm{c}}$, Behzat Sahin ${ }^{\mathrm{d}}$ \\ ${ }^{a}$ Department of Physics, Middle East Technical University, Ankara, 06800, Turkey \\ ${ }^{\mathrm{b}}$ Department of Physics, Bilkent University, Ankara, 06800 Turkey \\ ${ }^{\mathrm{c}}$ FiberLAST, Ankara, 06800, Turkey \\ ${ }^{\mathrm{d}}$ Department of Electronics and Communication Engineering, Yildirim Beyazit University, Ankara, \\ 06030, Turkey
}

\begin{abstract}
Repetition rate tuning enables the fast acquisition of $\mathrm{THz}$ pulse profiles [1]. By using this method we demonstrate a compact and broadband terahertz time domain spectroscopy system (THz TDS) driven by ytterbium doped fiber laser source. The importance of this method is realized in that $\mathrm{Yb}$ :doped fiber lasers can be amplified to sub-millijoule pulse strengths more easily than other types of fiber lasers [2]. Hence, it has the potential to be used in excite- $\mathrm{THz}$ probe experiments. Furthermore, the repetition rate-tuning adds flexibility in the excite-probe techniques. These attributes as well as $\mathrm{THz}$ generation and detection are investigated with the laser that was developed.
\end{abstract}

\section{INTRODUCTION AND BACKGROUND}

$\mathrm{T}$ ERAHERTZ time domain spectroscopy(THz-TDS) has proven to be one of the most productive tools in the farinfrared region $\left(10^{11}-10^{13} \mathrm{~Hz}\right)$ over the past two decades. While its dynamical nature allows excite-probe characterization studies with amplified lasers, costly and complex $\mathrm{THz}$ generation systems were a handicap. Typically, amplified Ti: $\mathrm{Al}_{2} \mathrm{O}_{3}$ systems operating near $800 \mathrm{~nm}$ are used in $\mathrm{THz}$ generation and detection in these methods. Increasing demands in THz TDS and field applications requires compact, portable and robust generation sources. Developing fiber laser technology enables us to produce ultrafast and powerful pulses that can be used in $\mathrm{THz}$ generation systems. Yb:doped fiber laser have greater potential in power scalability and robustness [3]. These ultrafast lasers with sub-200 femtosecond pulses enable scientists to use antenna structures, optical rectification, or surface emitters to produce $\mathrm{THz}$ [4]. Recent studies showed that $\mathrm{GaP}$ is an appropriate crystal for electro-optical rectification near $1 \mu \mathrm{m}$ region.

In this study, we present a compact THz-TDS driven by a robust $\mathrm{Yb}$-doped fiber laser operating at $50 \mathrm{MHz}$ repletion rate, $1035 \mathrm{~nm}$ central wavelength and sub-100fs pulse duration. A parallel line photoconductive antenna (PCA) is used as $\mathrm{THz}$ emitter and a $<110>$-cut GaP crystal is used for electro-optical sampling.

\section{RESULTS}

The fiber laser oscillator was tested for stability during the tuning of the repetition rate. The robust operation allowed for less than $3 \%$ drift of power over a tuning range of $60 \mathrm{kHz}$. After pre amplification stage (Figure 1) the oscillator will be incorporated in the $\mathrm{THz}$ system shown in Figure 2. The operation of the system will be analyzed in terms of stability, noise and overall implementation in an excite probe system which can be done after an additional amplification stage on the Yb:doped fiber laser. Consequently, this compact system can be used in pump-probe applications with high energy per pulse.

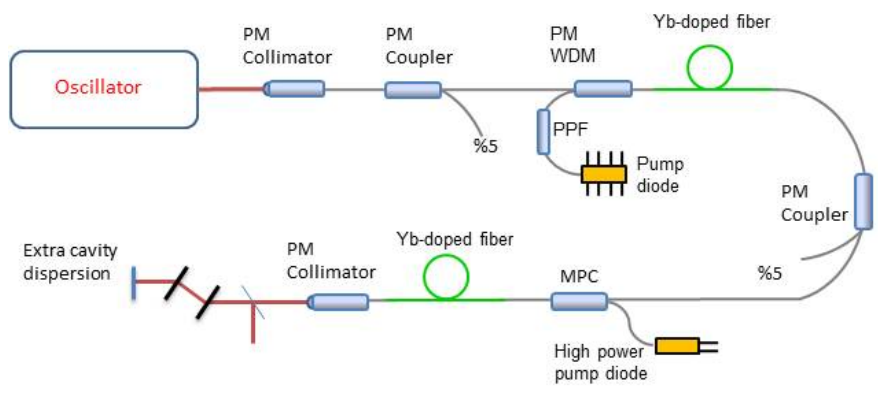

Figure 1: Yb-fiber laser figure,PM: Polarization maintaining, PPF: Pump protection filter, MPC: Multimode pump-signal combiner

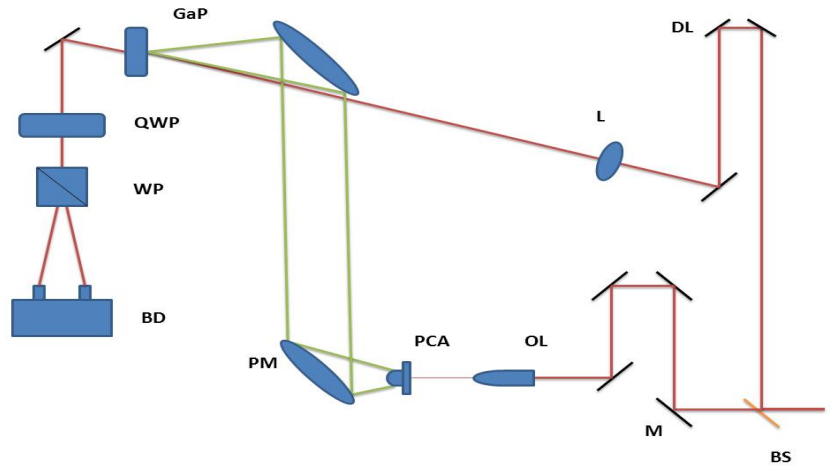

Figure 2: THz TDS system. quarter wave plate (QWP), Wollaston prism(WP), Objective lens (OL)

The Yb-doped fiber oscillator produces $\sim 30 \mathrm{~mW}$ power output. Central wavelength of oscillator is about $1040 \mathrm{~nm} 100$ fs pulses were derived from $\% 5$-monitoring output port. To implement the system in scheme as shown in Fig. 1, the stability of the oscillator was examined over the entire $\mathrm{THz}$ scan length by scanning the collimator over the range of 6 $\mathrm{mm}$. The measured repetition rate and power stability is plotted in Fig. 3. 

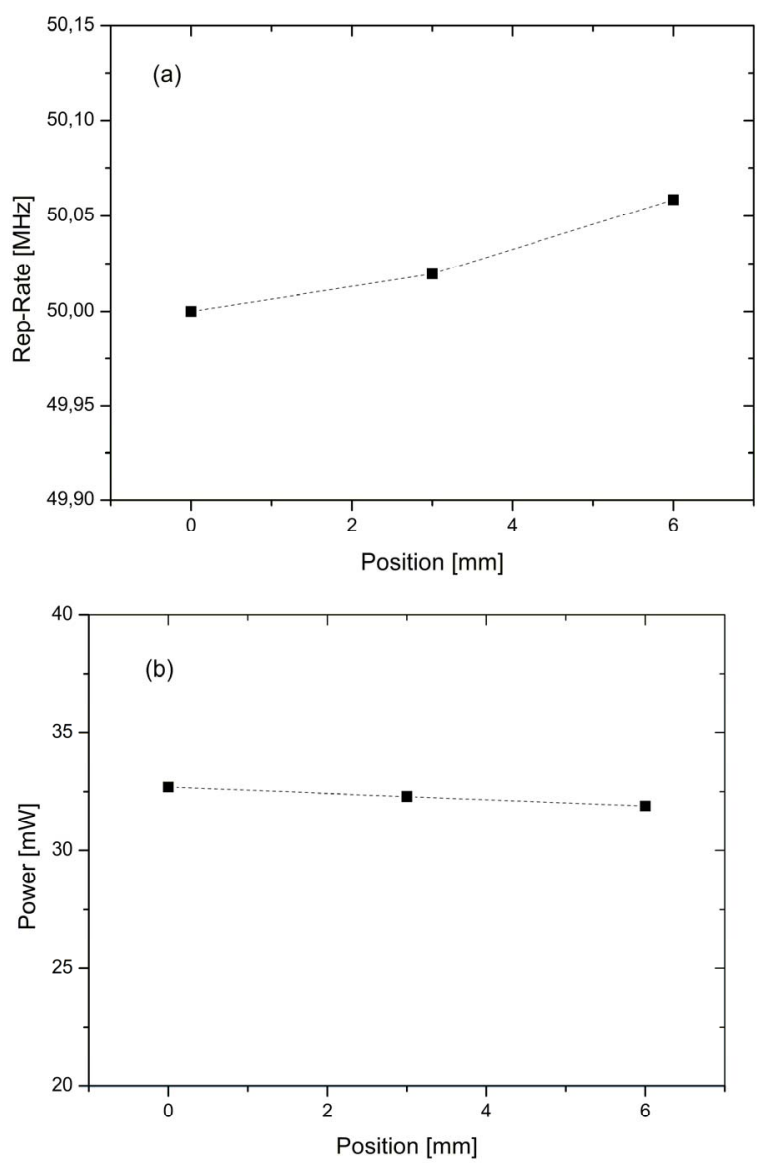

Figure 3 a Repetition rate change; b Power fluctuation as oscillator cavity is scanned

At the same time in order to observe stability of laser oscillator spectra were recorded during the scan interval. The obtained spectra show that the output has a center wavelength of about $1040 \mathrm{~nm}$ with a FWHM of $65 \mathrm{~nm}$ as shown in Fig 4a. Spectrum was observed to be stable with changing cavity length. Pulse durations for scan points are also measured as we scan $6 \mathrm{~mm}$ of cavity length. Autocorration (AC) signal of scanned pulses is show in Fig 4b. Intensity of oscillator output is normalized to 1 .

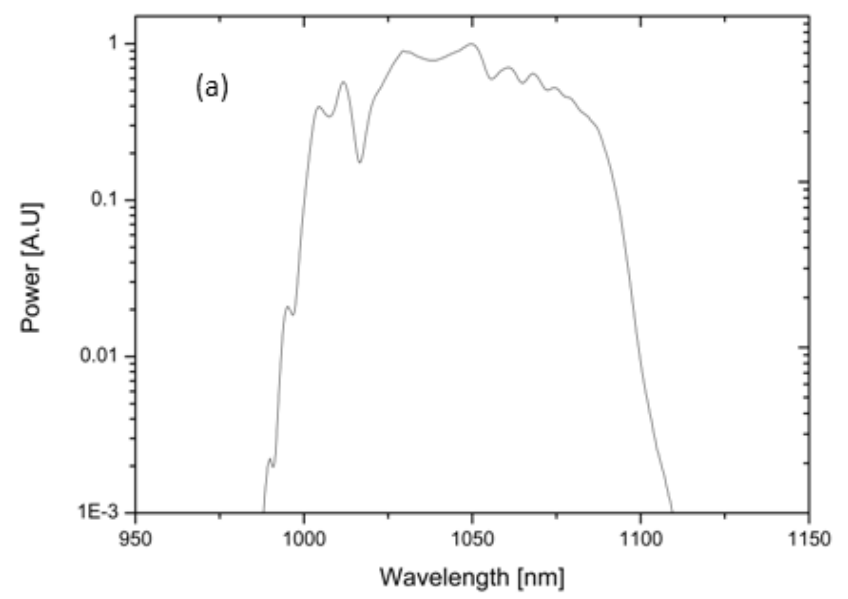

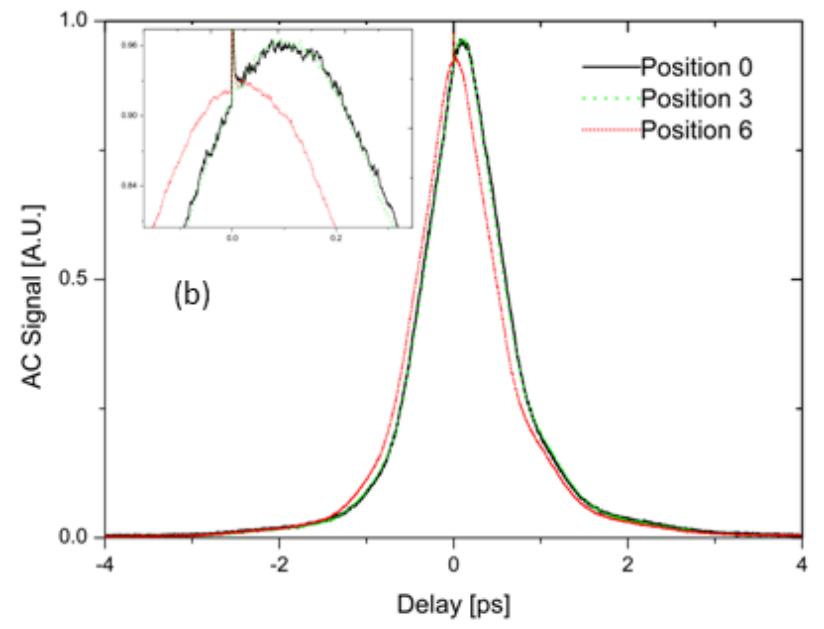

Fig. 4 a Spectrum output of oscillator; b Pulse profiles obtained during the scan length

Although there is some change at third position, the overall stability of the system is quite good with respect to expected measurements in the THz-TDS system. The pulse width change is below $1.01 \%$ and measured pulse durations of first, second and third positions are 965.05 fs, 969.47 fs and 974.78 fs respectively assuming pulse shape is Gaussian. This pulse will seed the amplifier stages and subsequently be compressed before being used in the $\mathrm{THz}$ generation and detection arms as shown in Fig. 1.

These results show that the oscillator can scan the $\mathrm{THz}$ waveform without the use of any external delay lines, thereby shortening the data acquisition times throughout the measurements. The oscillator for the amplified Yb-doped fiber laser system was constructed with a repetition rate of $50 \mathrm{MHz}$. In order to scan a length of $100 \mathrm{ps,} \mathrm{the} \mathrm{cavity} \mathrm{needs} \mathrm{to} \mathrm{be}$ tuned over $+/-25 \mathrm{kHz}$. Oscillator cavity is scanned over $6 \mathrm{~mm}$ range, for which the laser system exhibited little or no difference in output pulse duration, power and spectrum characteristics which is a testament to the stability of these lasers compared to other solid-state mode-locked lasers.

\section{ACKNOWLEDGMENTS}

This project was funded under TUBITAK Grant \# 111 T748

\section{References}

[1] Rafal Wilk, Thomas Hochrein, Martin Koch, Michael Mei, and Ronald Holzwarth, "Terahertz spectrometer operation by laser repetition frequency tuning," JOSA B, Vol. 28, Issue 4, pp. 592-595, 2011

[2] H. Kalaycioglu, K. Eken, and F. Ö. Ilday, “ Fiber amplification of pulse bursts up to $20 \mu \mathrm{J}$ pulse energy at $1 \mathrm{kHz}$ repetition rate," Optics Letters, Vol. 36, Issue 17, pp. 3383-3385, 2011

[3] Feng Liu, You-Jian Song, Qi-Rong Xing, Ming-Lie Hu, Yan-Feng Li, Chang-Lei Wang, Lu Chai, Wei-Li Zhang, Aleksei M. Zheltikov, and Ching-Yue Wang, "Broadband Terahertz Pulses Generated by a Compact Femtosecond Photonic Crystal Fiber Amplifier," IEEE Photon. Technol. Lett., vol. 22, No. 11, pp. 814-816, June 1, 2010.

[4] Guoqing Chang, Charles J. Divin, Chi-Hung Liu, Steven L. Williamson, Almantas Galvanauskas, and Theodore B. Norris, "Power scalable compact THz system based on an ultrafast Yb-doped fiber amplifier," Optics Express, Vol. 14, Issue 17, pp. 7909-7913, 2006 